${ }^{+}+{ }^{+}$La revue pour l'histoire du CNRS

POUR L'HISTOIRE DU CNRS

$17 \mid 2007$

Objectif biotechs?

\title{
Mémoires de mémoire
}

\section{Georges Chapouthier}

\section{OpenEdition}

Journals

Édition électronique

URL : https://journals.openedition.org/histoire-cnrs/2081

DOI : 10.4000/histoire-cnrs.2081

ISSN : 1955-2408

Éditeur

CNRS Éditions

Édition imprimée

Date de publication : 3 juillet 2007

ISBN : 978-2-271-06558-2

ISSN : 1298-9800

\section{Référence électronique}

Georges Chapouthier, " Mémoires de mémoire », La revue pour l'histoire du CNRS [En ligne], 17 | 2007. mis en ligne le 03 juillet 2009, consulté le 20 mai 2021. URL : http://journals.openedition.org/histoirecnrs/2081 ; DOI : https://doi.org/10.4000/histoire-cnrs.2081

Ce document a été généré automatiquement le 20 mai 2021.

Comité pour l'histoire du CNRS 


\title{
Mémoires de mémoire
}

\author{
Georges Chapouthier
}

1 Avant la deuxième guerre mondiale, des scientifiques comme Paul Guillaume ou Ignace Meyerson ont, les premiers, abordé le thème de la biologie de la mémoire. Mais ce type d'études a connu son apogée entre 1955 et 2000, qui s'est traduite par une abondance d'articles publiés et par l'importance conceptuelle des travaux effectués ${ }^{1}$.

2 Si le prix Nobel dans ce domaine a été attribué en 2000 à l'Américain Eric Kandel ${ }^{2}$, pour ses travaux sur la mémoire des aplysies, c'est bien en France, sous la direction de Ladislav Tauc, que cet illustre chercheur fait ses débuts. Dire l'importance de la recherche française, c'est souligner indirectement celle du CNRS, via ses agents ou les laboratoires qui bénéficiaient de fonds ou de financements ponctuels du CNRS. Le texte qui suit donne une liste non exhaustive des auteurs et de leurs travaux et présente une vision schématique d'une évolution de près de soixante années.

On ne parlera ici que de la biologie de la mémoire, principalement étudiée sur des modèles animaux, en négligeant volontairement d'autres domaines qui en sont proches et où ont été aussi publiés de nombreux travaux français: psychologie, pathologie neurologique, éthologie de terrain... Plusieurs pôles géographiques d'étude de la biologie de la mémoire ont joué un rôle primordial.

De Gif/Orsay...

4 Le pôle de Gif/Orsay comprend deux composantes originelles : le groupe d'étude de la mémoire chez les rongeurs animé par Vincent Bloch et le laboratoire de neurobiologie cellulaire dirigé par Ladislav Tauc. Vincent Bloch ${ }^{3}$ commença à s'intéresser, dès 1955 à Paris, à la "consolidation » de la mémoire, qui fait qu'un souvenir se stabilise et devient moins fragile. Il continua à développer cette thématique à Lille (1962) en la liant à l'activité d'une partie de l'encéphale, la "formation réticulaire activatrice», avant de constituer le pôle de Gif/Orsay. En quittant Lille en 1972 pour devenir professeur à Orsay, Bloch amena à Gif la plupart de ses élèves et recruta de nouveaux chercheurs. L'activité de groupe de Gif-sur-Yvette a porté sur l'évolution temporelle de la trace mnésique, avec les importants phénomènes de la « consolidation » ainsi que du «rappel» des souvenirs déjà consolidés (B. Deweer, J.-P. Lecanuet, P. Gisquet, N. Neuenschwander-El Massioui....). 
D'autres recherches du même groupe ont porté sur les relations entre la mémoire et le sommeil paradoxal, cette partie du sommeil où, paradoxalement, le cerveau est activé d'une manière analogue à ce qui se passe durant la veille et où se situe une large part de l'activité onirique (P. Leconte, E. Hennevin, B. Hars...) ainsi que sur la mise en évidence d'apprentissages cognitifs complexes chez le rat (T. Alexinsky, G. Chapouthier). L'activité du groupe perdura après le départ du laboratoire de Vincent Bloch pour Orsay (en 1992) et après sa succession par Serge Laroche en 1994. Ce dernier impulsa des recherches sur les phénomènes cellulaires de l'apprentissage dans le cerveau des mammifères. Le groupe contribua alors à l'analyse de la "potentialisation à long terme ", un phénomène de facilitation de l'activité synaptique qui pourrait être l'un des processus de base de la constitution des souvenirs.

Des chercheurs du groupe essaimèrent ailleurs : Jean-Pierre Lecanuet, spécialiste de la consolidation de l'empreinte chez le poussin, constitua un groupe de recherche sur l'embryon (humain) à l'université Paris V ; Martine Ammassari-Teule partit à Rome créer un laboratoire sur les rapports de la mémoire et de la génétique chez la souris; Susan Sara s'installa à Paris où elle montra notamment que le souvenir, même parfaitement consolidé et stabilisé, pouvait être rendu à nouveau à un état fragile de plasticité, lors du rappel, lorsque l'on se re-souvient de quelque chose, lorsque l'on réactive un souvenir ancien.

7 Une autre composante essentielle de ce pôle a été le laboratoire de neurobiologie cellulaire dirigé, à Gif-sur-Yvette, par Ladislav Tauc, où d'importants travaux furent effectués sur la mémoire des invertébrés, permettant ainsi de cerner des mécanismes de base de la mémoire sur des modèles simples, chez des êtres vivants moins élevés dans l'échelle phylogénétique de la complexité. Les premiers travaux de ce pôle ont été amorcés par Tauc, avec Jan Bruner, dès 1962 à Paris, à l'Institut Marey dirigé par Alfred Fessard. C'est là qu'Eric Kandel, futur prix Nobel, fit ses premières armes. Le laboratoire s'installa ensuite à Gif à partir de 1968 et y poursuivit ses recherches, notamment sur un apprentissage très simple de l'aplysie appelé l'» habituation » et qui fait qu'un stimulus répété plusieurs fois finit par ne plus déclencher une réponse: l'animal apprend à ne plus répondre (travaux de J. Bruner, J. S. Kehoe, T. Shimahara...). Un autre chercheur américain célèbre, Robert Hawkins, futur collaborateur d'Eric Kandel à New York, vint s'y former. À partir de 1995, le laboratoire poursuivit son activité sous la direction de Maurice Israël.

... à Bordeaux...

C'est autour d'abord de Bernard Cardo dès 1961, puis de son successeur Robert Jaffard à partir de 1988, que s'est constitué l'important pôle bordelais. Ce pôle s'est également intéressé à d'autres domaines, plus neurophysiologiques, du fonctionnement cérébral (M. Le Moal, L. Stinus ou P. Casala). Un axe essentiel en fut l'étude de la mémoire chez les rongeurs ${ }^{4}$, avec notamment les analyses du rôle de diverses régions cérébrales dans la consolidation (B. Soumireu-Mourat, R. Jaffard, C. Destrade). Les travaux portèrent d'abord sur le rat, puis, à l'initiative du prix Nobel François Jacob, elles s'orientèrent davantage vers la souris. À l'actif de ce groupe, l'implication d'une région du cerveau, appelée l'» hippocampe ", dans la mise en mémoire. Avec Robert Jaffard, le laboratoire s'intéressa ensuite à l'implication d'un médiateur cérébral dans l'apprentissage, l'»acétylcholine» (A. Marighetto). L'acétylcholine est le même médiateur dont on déplore la perte chez les patients (humains) atteints de la maladie d'Alzheimer. Dans le même temps, Daniel Béracochéa élaborait chez la souris un modèle reproduisant les 
troubles mnésiques dus à l'alcoolisme chez l'homme et Bruno Bontempi analysait la consolidation des souvenirs à très long terme.

À Bordeaux même, dans le laboratoire fondé par Michel Le Moal, Hervé Simon effectua chez le rat, en collaboration avec le neurologue Bruno Dubois, puis avec Willy Mayo, des recherches sur un noyau du tronc cérébral, dont on sait qu'il est impliqué, chez l'homme, dans les troubles de mémoire de la maladie d'Alzheimer. Tandis que Nora Abrous étudiait, par l'utilisation de greffes neuronales, les rapports de la mémoire avec la neurogenèse. Soumireu-Mourat partit fonder un groupe à Aix-Marseille où, avec son élève François Roman, il analysa les effets sur la mémoire de molécules hormonales. Le pôle bordelais eut également un rayonnement international. En témoignent les nombreuses collaborations des chercheurs bordelais (M. Le Moal, B. Bontempi) avec les laboratoires américains et l'arrivée de George Koob (USA) ou de Pier-Vincenzo Piazza (Italie).

... en passant par Strasbourg...

10 C'est à l'éthologiste Rémy Chauvin, succédant à Gaston Viaud à la tête du laboratoire de psychophysiologie, que l'on doit, dès 1963, le développement du pôle orienté vers les relations entre la mémoire, la synthèse de protéines cérébrales et le rôle de l'environnement (G. Chapouthier, A. Ungerer, B. Will...). Arielle Ungerer isola même à partir d'une plante, le Datura Stramonium, connue en médecine africaine, une molécule protéique très originale aux propriétés amnésiantes. René Misslin développa un groupe de recherche de psychopharmacologie sur les relations entre mémoire et facteurs émotionnels chez la souris. Les travaux de son élève, Catherine Belzung, devaient se poursuivre à Tours dans le domaine de l'anxiété des rongeurs. Bruno Will fonda un autre laboratoire, toujours à Strasbourg, où, avec Christian Kelche et JeanChristophe Cassel, furent développées des recherches sur les récupérations cognitives après des lésions cérébrales ${ }^{5}$, sur les processus attentionnels et sur les modifications biochimiques dans le cerveau des rongeurs âgés. Ces études sont d'un grand intérêt dans le cadre du vieillissement de la mémoire chez l'homme et de la maladie d'Alzheimer.

... et Lyon

11 En 1989, André Holley, qui travaillait sur la neurophysiologie de l'olfaction, lança un groupe d'études de la neurobiologie de la mémoire olfactive chez le rat (R. Gervais, N. Ravel, A. M. Mouly). Il contribua ${ }^{6}$ à montrer l'importance des structures olfactives, non seulement dans la réception des informations, mais aussi dans l'enregistrement d'une mémoire plus permanente, dans des réseaux nerveux, où l'information continue à circuler sous une forme dynamique, avec des manifestations repérables de l'activité bioélectrique. En parallèle à ce groupe qui travaillait sur l'animal, le groupe de JeanPierre Royet apporta des résultats similaires sur l'olfaction humaine.

D'autres lieux, d'autres équipes, d'autres travaux en France...

12 À Paris, l'équipe de Jean Delacour a pu montrer, chez le rat, que la lésion de noyaux d'une région du cerveau, le "thalamus ", conduisait à des amnésies spectaculaires des apprentissages spatiaux, que l'on pouvait rapprocher de certaines amnésies observées chez l'homme (notamment le syndrome dit «de Korsakoff»). À Clermont-Ferrand, Josette Alliot a analysé l'influence de molécules hormonales sur l'apprentissage. À Lyon, dans le laboratoire de Michel Jouvet consacré à l'étude du sommeil, Jean-Louis Valatx et ses collaborateurs ont précisé, chez les souris, les liens entre types de sommeil et apprentissage. Sur un versant plus éthologique, à Toulouse, ont été 
développés des apprentissages originaux pour une meilleure compréhension des capacités de mémoire chez les insectes : mouches drosophiles (J. Médioni, G. Vaysse) ou abeilles (M. Gauthier, M. Giurfa). Toujours chez les abeilles, plusieurs études de conditionnement furent effectuées, dans les années 1990, par Minha Pham, à la station de recherches apicoles de Bures-sur-Yvette. Enfin, rappelons les recherches de Thomas Préat, à Gif puis à Paris, sur les apprentissages à long terme chez la drosophile et leur rattachement à des aires particulières du cerveau de ces insectes. Chez les mollusques, à Caen, Raymond et Marie-Paule Chichery analysèrent les capacités d'apprentissage des seiches, cousines des pieuvres et particulièrement intelligentes.

... et à l'étranger

13 Ajoutons, pour conclure, l'impact international de travaux effectués hors de France par deux chercheurs formés à Paris. Le Suisse Daniel Bovet, prix Nobel en 1957 pour ses travaux sur les neuromédiateurs, démontra, en Italie, par la comparaison des performances de lignées de souris consanguines, l'existence de bases génétiques des aptitudes d'apprentissage 7. Le Français Georges Ungar proposa, aux États-Unis, une spectaculaire théorie moléculaire de la mémoire ${ }^{8}$. Même s'il s'agit là de travaux effectués à l'étranger, ils témoignent aussi, indirectement, de la vitalité de la recherche française de cette époque sur la biologie de la mémoire.

L'auteur souhaite remercier pour leurs conseils Vincent Bloch, Jan Bruner, Elisabeth Hennevin, Robert Jaffard, Jean-Marc Jallon, Christian Kelche, Nadine Ravel et Hervé Simon.

\section{NOTES}

1.Biologie de la mémoire. Chapouthier G. Odile Jacob, 2006.

2.Kandel E. The molecular biology of memory storage: a dialogue between genes and synapses. Science, 2001, 294(5544):1030-1038.

3.Psychologues de langue française. Collectif. Presses universitaires de France, 1992.

4.Jaffard R., Marighetto A. Neurobiologie de la mémoire. In Neurosciences et maladies du système nerveux. Korn H., (Sous la direction de). Lavoisier/Tec-et-Doc, 2003, pp. 95-110.

5.Will B., Kelche C., Cassel J.-C. Intracerebral transplants and memory dysfunction: circuitry repair or functional level setting?, Neural Plast, 2000, 7(1-2):93-108. Will B., Galani R., Kelche C., Rosenzweig M. R. Recovery from brain injury in animals: relative efficacy of environmental enrichment, physical exercise or formal training. Progress in Neurobiology of Learning and Memory, 2004, 72:167-182.

6.Martin C., Gervais R., Hugues E., Messaoudi B., Ravel N. Learning modulation of odorinduced oscillatory responses in the rat olfactory bulb: A correlate of odor recognition? Journal of Neuroscience, 2004, 24(2):389-397. Sevelinges Y., Gervais R., Messaoudi B., Granjon L., Mouly A. Olfactory fear conditioning induces field potential potentiation in rat olfactory cortex and amygdala. Learning and Memory, 2004, 11:761-769. 
7.Bovet D., Bovet-Nitti F., Oliverio A. Memory and consolidation mechanisms in avoidance learning of inbred mice. Brain Res., 1968, 10(2):168-182. Bovet D., Bovet-Nitti F., Oliverio A. Genetic aspect of learning and memory in mice. Science, 1969, 163:139-149.

8. Chapouthier G. Histoire de la neurochimie des processus mnésiques des origines à nos jours. In Histoires de la mémoire. Dupont J. C., (Sous la direction de). Vuibert, 2005, pp. 243-254.

\section{RÉSUMÉS}

Dans la seconde moitié du XX $\mathrm{X}^{\mathrm{e}}$ siècle, la recherche française, soutenue par le CNRS, a beaucoup produit dans le domaine de la biologie de la mémoire. Georges Chapouthier présente ici les grands courants et les principaux pôles géographiques.

In the second half of the $20^{\text {th }}$ century, research in France, backed by the CNRS, produced a great deal of work on the biology of memory. Georges Chapouthier has reviewed the major trends and listed the leading research centres.

\section{INDEX}

Mots-clés : biologie, pathologie, mémoire, biologie de la mémoire, maladie d'Alzheimer

\section{AUTEUR}

\section{GEORGES CHAPOUTHIER}

Docteur ès sciences en biologie et docteur ès lettres en philosophie, Georges Chapouthier est directeur de recherche au CNRS. Il est membre de l'unité CNRS «Vulnérabilité, adaptation et psychopathologie » et de l'Institut d'histoire et de philosophie des sciences et des techniques. 